\title{
3 Research Square \\ HER-3 Expression and MEK Activation in Non-small Cell Lung Cancer
}

\section{Thubeena Manickavasagar}

Royal Marsden NHS Foundation Trust https://orcid.org/0000-0002-5251-0403

\section{Wei Yuan}

Royal Marsden NHS Foundation Trust

\section{Suzanne Carreira}

Royal Marsden NHS Foundation Trust

\section{Bora Gurel}

Institute of Cancer Research

\section{Susana Miranda}

Royal Marsden NHS Foundation Trust

\section{Ana Ferreira}

Royal Marsden NHS Foundation Trust

\section{Mateus Crespo}

Royal Marsden NHS Foundation Trust

\section{Ruth Riisnaes}

Royal Marsden NHS Foundation Trust

\section{Chloe Baker}

Royal Marsden NHS Foundation Trust

\section{Mary O'Brien}

Royal Marsden NHS Foundation Trust

\section{Jaishree Bhosle}

Royal Marsden NHS Foundation Trust

\section{Sanjay Popat}

Royal Marsden NHS Foundation Trust

\section{Udai Banerji}

Royal Marsden NHS Foundation Trust

\section{Juanita Lopez}

Royal Marsden NHS Foundation Trust

Johann de Bono

Royal Marsden NHS Foundation Trust

Anna Minchom ( $\nabla$ anna.minchom@icr.ac.uk)

Royal Marsden NHS Foundation Trust https://orcid.org/0000-0002-9339-7101 
Research article

Keywords: NSCLC, HER-3, MEK, ERBB3

Posted Date: August 26th, 2020

DOI: https://doi.org/10.21203/rs.3.rs-59361/v1

License: (c) (i) This work is licensed under a Creative Commons Attribution 4.0 International License. Read Full License 


\section{Abstract}

Background

HER-3 has a role in pathogenesis and development of resistance to targeted therapies in non-small cell lung cancer (NSCLC).

Methods

We analysed tumour samples from 45 patients with adenocarcinoma of the lung. HER-3 and HER-2 expression was identified using immunohistochemistry as well as bioinformatic interrogation of The Cancer Genome Atlas (TGCA).

Results

HER-3 was highly expressed in $42.2 \%$ of cases and observed more frequently than HER-2 overexpression. ERBB3 copy number alone did not account for HER-3 overexpression. Bioinformatic analysis of TCGA demonstrated that MEK activity score (a score of downstream pathway activity and surrogate of functional HER-3 signalling) did not correlate with HER-3 ligands. EGFR pathway signalling was predominant with a significantly positive correlation of EGFR and $A R E G$ expression with MEK activity score. In the ERBB3 expressed group, ERBB3 RNA expression levels were significantly correlated with MEK activity score. ERBB3 expression level remained highly positively correlated with MEK activity after adjusting for EGFR expression.

Conclusion

HER-3 expression is common and it is not associated with gene amplification nor is it ligand dependent. HER-3 is an alternative pathway to EGFR activating MEK. HER-3 is a potential therapeutic target in NSCLC by virtue of frequent overexpression and functional downstream signalling.

\section{Background}

\section{Non-small cell lung cancer}

Lung cancer is the leading cause of cancer mortality worldwide. There is a poor 5 year survival rate in patients with regional and distant disease in non -small cell lung cancer (NSCLC), of $33 \%$ and $6 \%$ respectively (1). Systemic treatment with chemotherapy and immunotherapy is standard of care, while a small subset of cancers demonstrate oncogene-addiction with effective targeted treatments. These include EGFR mutated, ALK rearranged and newer targets such as ROS, RET and NTRK.

\section{HER-3}


HER-3 (receptor tyrosine-protein kinase erbB-3) is a member of the epidermal growth factor receptor (EGFR) family of receptors. Investigation of HER-3 as a therapeutic target has not been previously prioritised due to its impaired kinase activity (2). HER-3 acts as a heterodimeric partner for other members of the EGFR family, namely EGFR/HER-1 and ERB2/HER-2/Neu (3) (4) (5) (6). It has also been demonstrated that HER-3 can form heterodimers with MET, AXL and IGF1R (7) (8) (9). HER-3 possesses weak tyrosine kinase activity and so preferentially forms heterodimers with kinase- proficient receptor tyrosine kinases in the presence of its ligands neuregulin (NRG 1 and NRG 2) (2). EGFR has a number of ligands, including amphiregulin, epidermal growth factor and transforming growth factor, while HER-2 has no known ligand and remains in an 'open,' active conformation (10) (11) . However, HER-2/HER-3 dimerisation can occur independently of ligand binding (12). More lately it has been appreciated that HER-3 can also homodimerise on binding to the heregulin ligands, NRG1 and NRG2, so having intrinsic, non-EGFR or HER-2 mediated activity (13) (14). Downstream signalling of HER-3 is mainly via the PI3K/AKT pathway, with MAPK/ERK and JAK/STAT pathways also activated (15) (16).

ERBB3, (Erb-B Receptor Tyrosine Kinase 3) the HER-3 gene, is under the regulation of several miRNAs including miR125a, miR125b and miR205 (17) (18) (19). Studies have shown that the ubiquitinproteasome pathway plays a significant role in cancer initiation and progression by regulating protein levels (20) (21) (22) (23) . E3 ubiquitin ligases regulate EGFR family receptors. NRDP 1 mediates HER-3 degradation (24) (23). NRG1 stabilises USP8 which in turn stabilises NRDP1 (20) NEDD4 is a novel interaction partner and ubiquitin E3 ligase of HER-3 (21).

Evidence for the role of HER-3 in lung cancer pathogenesis is largely drawn from retrospective data sets correlating HER-3 expression with poor prognosis and metastatic development (25) (26) (27).

Accumulating evidence supports the role of HER-3 in the development of resistance to EGFR targeted therapies (28) (29) (30) (31). From preclinical data, dual targeting of EGFR and HER-3 is capable of overcoming acquired resistance to EGFR inhibition (29) (31). MET amplification or overexpression is a mechanism of resistance to EGFR-TKI occurring in $5 \%$ of cases (32). HER-3 phosphorylation is observed in EGFR mutant tumours with MET amplification, indicating that MET activates PI3K/AKT pathway via HER-3 phosphorylation (33). MEK inhibition causes a transcriptional upregulation of both HER-2 and HER-3 and the formation of heterodimeric complexes in KRAS mutant non-small cell lung cancer (NSCLC) and colon cancer and combined MEK and dual EGFR-HER-2 inhibition has a synergistic effect (30). Mutations of HER-3 have been described in the extra-cellular domain or tyrosine kinase domain as a rare event in lung cancer and can influence the activity of HER-3 receptor as well as the dimerization affinity with HER-2 and EGFR. (34) (35) (36).

Here, we explore HER-3 expression levels in adenocarcinoma of the lung (adeno-NSCLC) patients and correlate it with clinical parameters. We also interrogate large genomic datasets to identify the potential mechanism of HER-3 expression and propose that HER-3 could be an alternate pathway for MEK pathway activation.

\section{Methods}




\section{Patients and tissue samples}

The Drug Development Unit at the Royal Marsden (RMH) recruits patients with advanced solid malignancies to early phase clinical trials. Patient with adeno-NSCLC attending for consultation in our phase I clinic provided written informed consent and were enrolled into a tissue for research consent. Approval was gained from the local research and ethics committee for the use of the clinical data. Archival formalin fixed paraffin embedded tumour blocks were analysed for HER-2 and HER-3 protein expression and genomic analysis for HER-3 copy number was performed. Patient clinical data were retrospectively collected from the RMH electronic patient record system.

\section{Immunohistochemistry and scoring system}

Tissue blocks were freshly sectioned and only considered for immunohistochemistry (IHC) analyses of HER-2 and HER-3 if adequate material was present. Protein expression on $3 \mu \mathrm{m}$ thick FFPE sections was assessed by a Pathologist blinded to the clinical data.

HER-3 and HER-2 antibody validation by siRNA and immunohistochemical assays were performed as previously described.

Briefly, HER-3 immunoreactivity was investigated using the rabbit monoclonal anti-HER-3 antibody clone D22C5 (\#12708, Cell Signaling Technology, London, UK). HER-2 immunoreactivity was investigated using the mouse monoclonal anti-HER-2 antibody clone CB11 (\#NCL-L-CB11, Leica Biosystems, Newcastle, UK). Rabbit and Mouse IgGs (\#I-1000 and I-2000, Vector, Burlingame, CA, USA) were used as negative controls. For HER-3 IHC, antigen retrieval was conducted by heating slides in high pH buffer using a microwave, staining was subsequently performed using BioGenex 16000 autostainer (Launch Diagnostics; Kent; UK). HER-3 immunoreactivity was detected using Dako EnVision Flex high pH kit (\#K800021-2, Agilent; Glostrup; Denmark). For HER-2 immunohistochemistry automated antigen retrieval and detection were performed using Leica Bond RX (Leica Biosystems; Newcastle; UK). HER-2 immunoreactivity was detected using the Bond Polymer Refine Detection system (\#DS9800, Leica Biosystems; Newcastle; UK).

For HER-2, membranous expression was scored according to College of American Pathologists (CAP) HER-2 scoring guidelines (37). No staining or membrane staining that is incomplete and is faint/barely perceptible and in $\leq 10 \%$ of tumour cells (Score 0 ); incomplete membrane staining that is faint/ barely perceptible and in $>10 \%$ of tumour cells (Score +1 ); weak to moderate complete membrane staining observed in $>10 \%$ of tumour cells (Score $2+$ ) and circumferential membrane staining that is complete, intense and in $>10 \%$ of tumour cells (Score +3 ). For HER-3 staining, the same guidelines were used to generate an ad-hoc HER-2-like score for membranous staining. Additionally, HER-2 and HER-3 cytoplasmic staining was visually assessed, percentage of cells staining weakly, moderately or strongly were noted and $\mathrm{H}$ score calculated. 


\section{Digital PCR}

Digital polymerase chain reaction (PCR) was performed on a QX100 droplet digital PCR system (Bio-Rad). Copy Number Assays were performed with a FAM-ERBB3 validated Bio-Rad assay, reference ID: dHsaCP2500395 (67bp amplicon) and a recommended reference copy number Hex-assay for AP3B1, assay ID: dHsaCP2500348 (60bp amplicon). Digital PCR analysis was performed using QuantaSoft v1.3.2.0 software from Bio-Rad to assess the number of positive droplets. At least two negative control wells with no DNA were included in every run and each assay was run at least in duplicate (see Supplementary Methods).

\section{Statistical methods}

Statistical analysis of IHC and clinical data was performed on Graphpad Prism v7.0d. The association between HER-3 expression and other binary data was analysed using two sided Fishers exact test. For survival Kaplan-Meir curves were constructed and Gehan-Breslow-Wilcoxon test compared populations. Survival was taken from diagnosis to death and those alive at follow-up were censored.

\section{Bioinformatic methods}

Genomic data and gene expression (RSEM) for 507 adeno-NSCLC was downloaded from cBioportal for analysis (38). MEK activity score is an accumulation measurement of MEK pathway activity based on a previously validated 18-gene transcript signature of MEK signalling (39). The associations between gene expression levels (and with MEK signalling) were assessed by linear regression. All statistical test was performed using R (v1.1.43).

\section{Results}

\section{Clinical Characteristics}

From December 2010 to December 201845 patients with adeno-NSCLC were referred to the Drug Development Unit, consented to tissue analysis and had available archival tumour specimens. The majority of samples were from biopsies (41), the rest being from surgical resection (4). There were relatively fewer males (19) than females (26) in the cohort. The median age at diagnosis was 58 (range 38-72) and the median overall survival was 38 months. The median number of lines of treatment for advanced disease was 3 (range 0-7): 43 patients received chemotherapy (median 2, range 1-4), 22 patients received EGFR tyrosine kinase inhibitor (TKI) (median 1; range 1-3) and 9 patients received immunotherapy. All patients who had an EGFR mutation received an EGFR TKI and 25 patients were enrolled in a phase I trial and received at least 1 phase 1 trial drug.

\section{HER-2 and HER-3 protein expression}


HER-3 protein expression showed a membranous distribution with no nuclear expression (Fig. 1A). High expression of HER-3 ( $2+$ or $3+$ ) was seen in $42.2 \%$ of cases (Table 1 ). HER-2 expression was positive in $31.1 \%$ of cases, while HER-2 and HER-3 co-expression was seen in $11.1 \%(5 / 45)$. No correlation between HER-2 and HER-3 expression was demonstrated (Fishers exact test; $p>0.9999)$. Patient characteristics according to HER-3 status are described in Table 2.

Table 1

Membranous HER-3 and HER-2 protein expression by immunohistochemistry.

\begin{tabular}{|c|c|c|}
\hline HER-3 staining & $\%(n)$ & \\
\hline 0 & $35.5 \%(16)$ & $57.8 \%(26)$ \\
\hline 1 & $22.2 \%(10)$ & \\
\hline 2 & $15.5 \%(7)$ & $42.2 \%(19)$ \\
\hline 3 & $26.7 \%(12)$ & \\
\hline HER-2 staining & $\%(n)$ & \\
\hline 0 & $33.3 \%(15)$ & $68.9 \%(31)$ \\
\hline 1 & $35.5 \%(16)$ & \\
\hline 2 & $22.2 \%(10)$ & $31.1 \%(14)$ \\
\hline 3 & $8.8 \%(4)$ & \\
\hline
\end{tabular}


Table 2

Clinical characteristics for HER-3 positive and HER-3 negative patients

\begin{tabular}{|lll|}
\hline Clinical Characteristics & HER-3 positive \% (n) & HER-3 negative \% (n) \\
\hline Male & $26 \%(5)$ & $74 \%(14)$ \\
\hline Female & $54 \%(14)$ & $46 \%(12)$ \\
\hline Median Age (years) & 58 & 57.5 \\
\hline Smoking status & & $12 \%(3)$ \\
\hline Non smoker & $16 \%(3)$ & $12 \%(3)$ \\
\hline Current smoker & $5 \%(1)$ & $35 \%(9)$ \\
\hline Ex-smoker & $47 \%(9)$ & $42 \%(11)$ \\
\hline Unknown & $32 \%(6)$ & $2 \%(1)$ \\
\hline Prior therapy & & $43 \%(25)$ \\
\hline No prior treatment & $2 \%(1)$ & $7 \%(4)$ \\
\hline Prior chemotherapy & $35 \%(17)$ & $22 \%(13)$ \\
\hline Prior immunotherapy & $10 \%(5)$ & $26 \%(15)$ \\
\hline Prior TKI & $23 \%(11)$ & \\
\hline Phase 1 Clinical Trial & $17 \%(10)$ & \\
\hline TKl: tyrosine kinase inhibitor & \\
\hline
\end{tabular}

\section{Correlation of HER-3 protein expression by IHC with clinical characteristics}

The median overall survival from diagnosis for HER-3 positive patients was 40.5 months, and for HER-3 negative patients 30.8 months. There was no statistical difference in survival between HER-3 positive and HER-3 negative cases (Gehan-Breslow-Wilcoxon; $p=0.4596)$ (Fig. 1B). There was no difference in median survival between HER-3 positive and negative cases in those patients with EGFR mutations (median OS 42.6 months HER-3 positive, median OS 43.9 months HER-3 negative; Gehan-Breslow-Wilcoxon; $p=$ 0.4927).

\section{HER-3 gene amplification}


Digital PCR of ERBB3 was performed in 27 cases. There was low frequency of small copy number gain in HER-3 with 11\% (3/27) having copy number variant (CNV) of 3-4 (Fig. 1C). Six out of the seven samples with this highest CNV scored $3+$ on HER-3 immunohistochemistry.

EGFR and KRAS mutations and correlation with HER-3

As standard of care EGFR mutational analysis had been performed in all 45 patients and KRAS mutational analysis in 28 patients. EGFR mutation was present in $17.8 \%$ ( 8 cases). HER-3 protein overexpression was present in $8.9 \%$ (4 cases) of EGFR mutation and in $14.2 \%$ (4 cases) of KRAS mutation. There was no correlation between HER-3 overexpression and EGFR positivity (Fishers exact test $p=0.7043$ ) or HER-3 and KRAS positivity (Fishers exact test; $p=0.6908$ ) (Fig. 2).

\section{Baseline genomic data in TCGA}

Interrogation of the PanCancer TCGA cancer genome atlas of 507 patients with adeno-NSCLC showed a genetic alteration rate of $31 \%$ in the ERBB family with the most common genetic alteration seen in EGFR and ERBB4, which was evident in 16\% (79 cases) and 10\% (50 cases). In ERBB2 and ERBB3, genetic alterations were less frequent; seen in $4 \%$ (19 cases) and 5\% (25 cases) respectively (Fig. 3A). Among the different types of genetic alteration, amplification was the most common alteration in $E R B B 3$, observed in $48 \%$ out of all alterations (12 cases). ERBB3 copy number change demonstrated a positive association with ERBB3 RNA expression ( $\left.r=0.26 ; p=8 \times 10^{-6}\right)$ (Fig. 3B); amplification cases had 1.7 times higher expression of ERBB3 RNA than diploid cases $(p=0.004)$.

ERBB3 amplification co-occurred with EGFR amplification; the amplification of ERBB3 was four times more likely to co-exist with EGFR amplification than not, though this did not reach statistical significance $(p=0.1)$. There was no co-amplification between $E R B B 3$ and $E R B B 2$, but the expression of ERBB3 was highly correlated with ERBB2 expression levels $\left(r=0.09\right.$ and $\left.p=2.5 \times 10^{-23}\right)$ (Figure S1). ERBB3 expression also positively correlates with ERBB4 expression ( $r=0.1$ and $p=0.001$ ) but there was no correlation with the expression levels of EGFR or ( $r=-0.12$ and $p=0.5)$.

\section{MEK activity score correlation with key nodes in ERBB pathway in TCGA data}

We performed further analyses of RNA sequencing data from TCGA. We observed ERBB2 and ERBB3 RNA expression levels were higher than EGFR and ERBB4 in adeno-NSCLC (Fig. 3C). We measured absolute gene expression level of $E R B B$ family genes and derived MEK pathway activity using a validated 18-gene MEK signature (39). EGFR RNA expression levels correlated with MEK activity $(r=0.16$ and $p=$ $\left.3.0 \times 10^{-5}\right), E R B B 3$ RNA expression did not correlate with MEK activity $(r=0.09$ and $p=0.78)$ (Fig. 3D). We further characterised MEK activation score with gene expression levels of ERBB ligand and proteins of ubiquitination (Table 3). AREG (EGFR ligand) and RNF41 (an ubiquitin ligase) had a significant positive correlation with MEK activity score. 
Table 3

Correlation of MEK activity score with expression of genes of interest.

\begin{tabular}{|lll|}
\hline Gene & Fvalue of correlation & $p$-value for correlation \\
\hline EGFR & 0.16031096 & $3.04 \mathrm{E}-05$ \\
\hline ERBB2 & -0.0453439 & 0.73555119 \\
\hline ERBB4 & 0.08977428 & 0.21373994 \\
\hline AREG & 0.27539042 & $1.68 \mathrm{E}-23$ \\
\hline NRG2 & -0.1101635 & 0.96120646 \\
\hline NRG1 & -0.0513447 & 0.8346032 \\
\hline HRG & -0.0578008 & 0.08479387 \\
\hline NEDD1 & -0.0384113 & 0.36325262 \\
\hline RNF41 & 0.13750166 & 0.0009244 \\
\hline USP8 & 0.03146702 & 0.52317997 \\
\hline $\begin{array}{l}\text { EGFR; epidermal growth factor, AREG: amphiregulin, NRG2: neuregulin 2, NRG1: neuregulin 1, HRG: } \\
\text { heregulin, NEDD1: neural precursor expressed, developmentally down-regulated protein1, RNF41: ring } \\
\text { finger 41, USP8: ubiquitin-specific protease 8 }\end{array}$ \\
\hline
\end{tabular}

In order to stratify ERBB3 RNA expression effects on MEK activity, we grouped patients into ERBB3 "expression high" (higher 50\% of ERBB3 RNA expression cases) and ERBB3 "expression low" (lower 50\% of ERBB3 RNA expression cases). In the ERBB3 expression high group, ERBB3 RNA expression levels were significant and positively correlated with MEK activity score $(r=0.19, p=0.006)$, whereas no correlation was observed in the ERBB3 expression low group ( $p=0.12)$ (Fig. 4). The expression of EGFR did not correlate with MEK activity score in the ERBB3 expression high group $(p=0.11)$, though did correlate with MEK activity score in the ERBB3 expression low group $\left(r=0.2, p=4 \times 10^{-5}\right)$. In the ERBB3 expression high group, ERBB3 expression levels still highly positively correlated with MEK activity even after adjusting for EGFR expression effects $(p=0.007)$.

\section{Discussion}

\section{HER-3 protein levels in adeno-NSCLC and mechanism of overexpression}

Reports of the incidence of HER-3 protein overexpression in NSCLC vary greatly according to the cohort. Previous groups have reported rates of $6.5 \%$ (NSCLC) (40), 15.9\% (advanced adeno-NSCLC) (27), 35\% (EGFR mutant NSCLC) (41), 64\% (stage 1 NSCCL) (42), 85.1\% (resected stage I-IIIA adeno-NSCLC) (43) 
and $86.1 \%$ (resected stage 0 and $1 \mathrm{~A} 1$ adeno-NSCLC) (44). IHC methods and scoring have not been previously standardised. Our data uses a validated and robust scoring system based on optical density and $\mathrm{H}$-score. Although our patient cohort is small, we demonstrate that overexpression of HER-3 protein is common in advanced adeno-NSCLC, occurring in over $40 \%$ of patients.

The rate of ERBB3 gene amplification in NSCLC is low. The Cancer Genome Atlas Research Group sequenced 230 cases of lung adenocarcinoma and did not identify any ERBB3 amplification (45). The cBioportal and GENIE data sets report amplification in just over 1\% frequency (38). A smaller series of 71 cases reported a $1 \%$ amplification rate in lung adenocarcinoma (34). In a series of 82 NSCLC patients HER-3 amplification was identified in $26.8 \%$ of patients who were treated with the EGFR TKI gefitinib. $E R B B 3$ amplification was more common in those with ERBB2 gain $(p=0.055)$ and EGFR gain $(p=0.06)$ but not with EGFR mutations ( $p=0.17$ ) (46). It was not possible to perform ERBB3 PCR on all cases in our cohort as tumour tissue was limited, but a sample of 27 cases had sufficient tumour sample to pass quality control. There appeared to be clustering of HER-3 overexpressing cases in those with ERBB3 copy number gain but this does not account for the majority of HER-3 overexpression.

Bioinformatic analysis of large, publicly available datasets allows testing of hypotheses. We interrogated the PanCancer TCGA data set of adeno-NSCLC samples. This dataset includes transcriptome (RNA) and genomic (DNA) data. The genomic data supports our finding of low levels of ERBB3 amplification, which was the most common cause of ERBB3 genetic alteration. It also provided the confirmation that $E R B B 3$ copy number alteration highly associates with its gene expression.

The MEK activity score is an 18 gene signature derived from an experiment to test a mixed-tumour response to the MEK inhibitor, selumetinib. The MEK activity score is a surrogate marker of MEK pathway activation. It is not specific for activation through the HER-3 channel as the MEK pathway is also initiated upon HER-2, EGFR and other receptor activation. It also does not take into account HER-3 downstream activation via the PIK3A/AKT/mTOR pathway. However, as HER-3 activity is predominantly via HER2/HER-3 and EGFR/HER-3 hetero-dimerisation, we inferred that the MEK activity score could be used as a surrogate of, but not specific to, HER-3 protein expression. If a key node in the HER-3 pathway is found to correlate with MEK activity score it can be concluded that, in that cohort, the node may play a key part in pathway. The lack of correlation with ERBB3 ligands (NRG 1 and 2) expression is an important negative finding. RNF41 also had a significant positive correlation with MEK activity score. This is difficult to interpret given the fact that an increase in a degradation protein would be expected to correlate with a lower target protein level.

In conclusion, HER-3 protein is common in adeno-NSCLC, amplification levels of ERBB3 are low and on TCGA analysis the mechanism of HER-3 protein overexpression does not appear to be ERBB3 ligand dependent. Further investigation is required to elucidate the mechanism of HER-3 protein overexpression in adeno-NSCLC.

\section{HER-3 expression as a prognostic marker}


Yi et $a^{26}$ demonstrated that HER-3 overexpression is associated with a poor prognosis in a cohort of 443 patients with advanced NSCLC (27). The median overall survival in our study was not significantly different between the HER-3 negative and positive patient group. This phase 1 patient cohort may not be truly reflective of the broader population of NSCLC patients, as these patients tend to be fitter with fewer or no co-morbidities.

\section{HER-3 in relation to oncogenic drivers in adeno-NSCLC}

HER-2 protein overexpression is reported in $6-30 \%$ of patients with lung cancer, which in keeping with our results (47) (48) (49). HER-2 expression is less frequently observed in NSCLC than HER-3 expression. There was some overlap, though no statistically significant association between, HER-3 expression with HER-2 expression or KRAS and EGFR mutations. Previous data have demonstrated that HER-3 plays a role in EGFR TKI resistance. Half of the cases in this cohort with EGFR mutation also co-expressed HER-3. No difference in overall survival was seen between HER-3 expressing and non-expressing groups in the EGFR-mutant cases in this cohort though patient numbers were small.

We therefore conclude that HER-3 represents a common target in NSCLC independent of KRAS and EGFR mutations. We have a simple tool in the form of IHC that can be used to "pre-screen" and stratify patients to receive such HER-3 targeting compounds. Furthermore, there are some patients in which HER-3 expression occurs concurrently with driver mutations and the role of HER-3 in these groups, including in drug resistance, requires further investigation. The relationship between HER-3 expression and other drivers in NSCLC (such as ROS, ALK, NTRK and RET) is yet to be determined.

\section{HER-3 and functional activity in adeno-NSCLC}

In the TCGA dataset MEK activity score was associated with EGFR and AREG (EGFR ligand) gene expression. This suggests that signalling via EGFR is key to the oncogenic behaviour in lung cancer. EGFR has been previously reported as a key driver of oncogenesis in lung (and other) cancers (50). Although our initial analysis did not show correlation of ERBB3 with MEK signature, we stratified ERBB3 expression into two groups; "expression low" and "expression high"; and analysed this along with EGFR gene expression. In the ERBB3 expression high group, ERBB3 RNA expression levels positively correlated with MEK activity score but not in the expression low group. The expression of EGFR correlated with MEK activity score in the ERBB3 expression low group. This supports the hypothesis that HER-3 is an alternative pathway for MEK activation in the HER-3 expression high group. This analysis implies a degree of functional downstream pathway signalling via HER-3. In effect, this suggests that when EGFR drives the cancer, the effect of HER-3 is minimal as EGFR drives the downstream signalling. However, as HER-3 expression increases this becomes the main driver of pathway activation. This finding has relevance in terms of proposing HER-3 as a viable drug target in adeno-NSCLC. As a membrane target HER-3 can be potentially targeted with drugs such as HER-3 target antibody drug conjugates in which expression on the surface is key to activity. However, a degree of functional MEK pathway activation 
suggests that in some patients drugs that rely on abrogation of signalling (such as HER-3/ HER-2 dimerisation drugs) can also be attempted. The challenge in this patient cohort is to predict patients in which this approach will be effective, our data relies on gene expression but transcriptome analysis is complex in a clinical trial setting.

\section{Conclusion}

In conclusion, HER-3 protein is commonly overexpressed in adeno-NSCLC though gene amplification levels are low. In the search for non-genomic causes of HER-3 protein overexpression, the MEK activity score was used as a surrogate of HER-3 overexpression; however no correlation with ligands of HER-3 were seen. Further investigation is therefore needed to continue to elucidate the mechanism of HER-3 overexpression. In adeno-NSCLC, signalling via the EGFR pathway is predominant but in the HER-3 expression high group HER-3 is an alternative pathway, with downstream signalling via the MEK pathway. Since HER-3 overexpression is more common than previously thought and appears to be functional in non-EGFR driven adeno-NSCLC, its utility as a therapeutic target in adeno-NSCLC should be a drug development priority.

\section{List Of Abbreviations}


Adeno-NSCLC Adenocarcinoma of the lung

\begin{tabular}{|c|c|}
\hline AKT & Protein kinase B \\
\hline ALK & Anaplastic lymphoma kinase \\
\hline AREG & Amphiregulin \\
\hline$A X L$ & Tyrosine-protein kinase receptor UFO \\
\hline CAP & College of American Pathologists \\
\hline CNV & Copy number variant \\
\hline EGFR & Epidermal growth factor receptor \\
\hline ERBB3 & Erb-B Receptor Tyrosine Kinase 3 \\
\hline ERBB4 & Erb-B Receptor Tyrosine Kinase 4 \\
\hline ERK & Extracellular Receptor Kinase \\
\hline FFPE & Fromalin fixed paraffin embedded \\
\hline HER-1 & Receptor tyrosine- protein kinase erbB-1 \\
\hline HER-2 & Receptor tyrosine- protein kinase erbB-2 \\
\hline HER-3 & Receptor tyrosine- protein kinase erbB-3 \\
\hline IGF-1 & Insulin- like growth factor 1 \\
\hline IGF1R & Insulin-like growth factor 1 receptor \\
\hline $\mathrm{IHC}$ & Immunohistochemistry \\
\hline JAK & Janus Kinase \\
\hline KRAS & Kirsten rat sarcoma \\
\hline MAPK & Mitogen activated protein kinase \\
\hline MEK & Mitogen-activated protein kinase kinase 1 \\
\hline mTOR & Mammalian target of rapamycin \\
\hline NEDD4 & Neural precursor expressed 4 \\
\hline NRDP1 & Neuregulin receptor degradation protein- 1 \\
\hline NRG-1 & Neuregulin 1 \\
\hline NRG-2 & Neuregulin 2 \\
\hline NSCLC & Non small cell lung cancer \\
\hline NTRK & Neurotrophic tropomyosin-related kinase \\
\hline
\end{tabular}




\begin{tabular}{ll} 
PCR & Polymerase chain reaction \\
\hline PI3K & Phosphatidylinositol 3-kinase \\
\hline RET & Rearranged during Transfection tyrosine kinase \\
\hline RMH & Royal Marsden Hospital \\
\hline RNF41 & Ring Finger Protein 41 \\
\hline ROS & C-ros proto-oncogene \\
\hline siRNA & Small interfering RNA \\
\hline STAT & Signal Transducer and Activator of Transcription. \\
\hline TGCA & The Cancer Genome Atlas \\
\hline TKI & Tyrosine kinase inhibitors \\
\hline USP8 & Ubiquitin-specific protease 8 \\
\hline
\end{tabular}

\section{Declarations}

\section{Ethical approval and Consent to participate}

Approval was obtained from the Royal Marsden Committee for Clinical Research (ref SE808). Written informed consent was obtained from participants prior to obtaining their archival tissue for research.

\section{Consent for publication}

Not applicable

\section{Availability of supporting data}

The datasets supporting the conclusions of this article are available in the within the article and its supplementary materials. Some of these data were derived from the following resources available in the public domain: The Cancer Genome Atlas https://portal.gdc.cancer.gov/projects.

\section{Competing interests}




\section{T \\ Manickavasagar}

\begin{tabular}{ll}
\hline W Yuan & None declared \\
\hline S Carreira & None declared \\
\hline B Gurel & None declared \\
\hline S Miranda & None declared \\
\hline A Ferreira & None declared \\
\hline M Crespo & None declared \\
\hline R Riisnaes & None declared \\
\hline C Baker & None declared \\
\hline MER O'Brien & Advisory boards for MSD, Abbvie, BMS, BI, Pierre Fabre \\
& All unrelated to this work \\
\hline J Bhosle & None declared \\
\hline S Popat & $\begin{array}{l}\text { Honoraria from BMS, Roche, Takeda, AstraZeneca, Pfizer, MSD, EMD Serono, } \\
\text { Guardant Health, AbbVie, Boehringer Ingelheim, Medscape, Tesaro, Paradox, } \\
\text { Incyte, OncLive } \\
\text { Direct funding: Elsevier } \\
\text { All unrelated to this work }\end{array}$ \\
\hline
\end{tabular}

U Banerji

Has received honoraria fron Astellas, Novartis, Karus Therapuetics, Pheonix Solutions, Eli Lilly, Astex, Vernalis, Boehringer Ingelheim

Is a recipient of an NIHR Research Professorship Award and has received CRUK funding: Cancer Research UK Scientific Executive Board, Cancer Research UK Centre Award. Cancer Research UK Drug Discovery Committee - Programme Award.

\section{All unrelated to this work}

J Lopez

$\mathrm{J}$ de Bono

\section{Research grant funding from Roche, Basilea, and Genmab unrelated to this work}

Has served on advisory boards and received fees from many companies including Astra Zeneca, Astellas, Bayer, Boehringer Ingelheim, Cellcentric, Daiichi, Genentech/Roche, Genmab, GSK, Janssen, Merck Serono, Merck Sharp \& Dohme, Menarini/Silicon Biosystems, Orion, Pfizer, Qiagen, Sanofi Aventis, Sierra Oncology, Taiho, Vertex Pharmaceuticals. He is an employee of The ICR, which have received funding or other support for his research work from AZ, Astellas, Bayer, Cellcentric, Daiichi, Genentech, Genmab, GSK, Janssen, Merck Serono, MSD, Menarini/Silicon Biosystems, Orion, Sanofi Aventis, Sierra Oncology, Taiho, , Pfizer, Vertex, and which has a commercial interest in abiraterone, PARP inhibition in DNA repair defective cancers and PI3K/AKT pathway inhibitors (no personal income). He was named as an inventor, with no financial interest, for patent $8,822,438$. He has been the $\mathrm{Cl} / \mathrm{PI}$ of many industry sponsored clinical trials. JDB is a National Institute for Health Research (NIHR) Senior Investigator. 
All unrelated to this work

A Minchom Has served on advisory boards and received fees from Merck, FARON, Novartis, Bayer, Janssen

All unrelated to this work

\section{Funding}

This study represents independent research supported by the National Institute for Health Research (NIHR) Biomedical Research Centre at the Royal Marsden NHS Foundation Trust and the Institute of Cancer Research. The views expressed are those of the authors and not necessarily those of the NIHR or the Department of Health and Social Care.

The authors also acknowledge funding from Cancer Research UK and the Experimental Cancer Centre Initiative.

\section{Authors' contribution}

All authors read and approved the final manuscript. 


\begin{tabular}{ll} 
TM & Data interpretation, drafted work \\
\hline WY & Data analysis, data interpretation, drafted work \\
\hline SC & Data analysis, data interpretation, revised work \\
\hline BG & Data analysis, data interpretation, revised work \\
\hline SM & Data analysis, data interpretation, revised work \\
\hline AF & Data analysis, revised work \\
\hline MC & Data analysis, revised work \\
\hline RR & Data analysis, revised work \\
\hline CB & Data analysis, revised work \\
\hline MERO & Revised work \\
\hline JB & Revised work \\
\hline SP & Revised work \\
\hline UB & Revised work \\
\hline JL & Revised work \\
\hline JdB & Revised work \\
\hline AM & Data interpretation, drafted work \\
\hline
\end{tabular}

\section{Acknowledgements}

Nil

\section{Authors' information}

Nil

\section{References}

1. American Cancer Society. American Cancer Society. Lung Cancer Survival Rates 2019 [Available from: https://www.cancer.org/cancer/lung-cancer/detection-diagnosis-staging/survival-rates.html.

2. Shi F, Telesco SE, Liu Y, Radhakrishnan R, Lemmon MA. ErbB3/HER3 intracellular domain is competent to bind ATP and catalyze autophosphorylation. Proc Natl Acad Sci U S A. 2010;107(17):7692-7. 
3. Wallasch C, Weiss FU, Niederfellner G, Jallal B, Issing W, Ullrich A. Heregulin-dependent regulation of HER2/neu oncogenic signaling by heterodimerization with HER3. EMBO J. 1995;14(17):4267-75.

4. Pinkas-Kramarski R, Soussan L, Waterman H, Levkowitz G, Alroy I, Klapper L, et al. Diversification of Neu differentiation factor and epidermal growth factor signaling by combinatorial receptor interactions. EMBO J. 1996;15(10):2452-67.

5. Sergina NV, Rausch M, Wang D, Blair J, Hann B, Shokat KM, et al. Escape from HER-family tyrosine kinase inhibitor therapy by the kinase-inactive HER3. Nature. 2007;445(7126):437-41.

6. Engelman JA, Janne PA, Mermel C, Pearlberg J, Mukohara T, Fleet C, et al. ErbB-3 mediates phosphoinositide 3-kinase activity in gefitinib-sensitive non-small cell lung cancer cell lines. Proc Natl Acad Sci U S A. 2005;102(10):3788-93.

7. Tanizaki J, Okamoto I, Sakai K, Nakagawa K. Differential roles of trans-phosphorylated EGFR, HER2, HER3, and RET as heterodimerisation partners of MET in lung cancer with MET amplification. $\mathrm{Br} J$ Cancer. 2011;105(6):807-13.

8. Revach OY, Sandler O, Samuels Y, Geiger B. Cross-Talk between Receptor Tyrosine Kinases AXL and ERBB3 Regulates Invadopodia Formation in Melanoma Cells. Cancer Res. 2019;79(10):2634-48.

9. Chen C, Gupta P, Parashar D, Nair GG, George J, Geethadevi A, et al. ERBB3-induced furin promotes the progression and metastasis of ovarian cancer via the IGF1R/STAT3 signaling axis. Oncogene. 2020;39(14):2921-33.

10. Cho HS, Mason K, Ramyar KX, Stanley AM, Gabelli SB, Denney DW Jr, et al. Structure of the extracellular region of HER2 alone and in complex with the Herceptin Fab. Nature. 2003;421(6924):756-60.

11. Garrett TP, McKern NM, Lou M, Elleman TC, Adams TE, Lovrecz GO, et al. The crystal structure of a truncated ErbB2 ectodomain reveals an active conformation, poised to interact with other ErbB receptors. Mol Cell. 2003;11(2):495-505.

12. Lee-Hoeflich ST, Crocker L, Yao E, Pham T, Munroe X, Hoeflich KP, et al. A central role for HER3 in HER2-amplified breast cancer: implications for targeted therapy. Cancer Res. 2008;68(14):5878-87.

13. Steinkamp MP, Low-Nam ST, Yang S, Lidke KA, Lidke DS, Wilson BS. erbB3 is an active tyrosine kinase capable of homo- and heterointeractions. Mol Cell Biol. 2014;34(6):965-77.

14. Waterman H, Sabanai I, Geiger B, Yarden Y. Alternative intracellular routing of ErbB receptors may determine signaling potency. J Biol Chem. 1998;273(22):13819-27.

15. Hellyer NJ, Cheng K, Koland JG. ErbB3 (HER3) interaction with the p85 regulatory subunit of phosphoinositide 3-kinase. Biochem J. 1998;333(Pt 3):757-63.

16. Vijapurkar U, Cheng K, Koland JG. Mutation of a Shc binding site tyrosine residue in ErbB3/HER3 blocks heregulin-dependent activation of mitogen-activated protein kinase. J Biol Chem. 1998;273(33):20996-1002.

17. Wang Z, Liao H, Deng Z, Yang P, Du N, Zhanng Y, et al. miRNA-205 affects infiltration and metastasis of breast cancer. Biochem Biophys Res Commun. 2013;441(1):139-43. 
18. Iorio MV, Casalini P, Piovan C, Di Leva G, Merlo A, Triulzi T, et al. microRNA-205 regulates HER3 in human breast cancer. Cancer Res. 2009;69(6):2195-200.

19. Scott GK, Goga A, Bhaumik D, Berger CE, Sullivan CS, Benz CC. Coordinate suppression of ERBB2 and ERBB3 by enforced expression of micro-RNA miR-125a or miR-125b. J Biol Chem. 2007;282(2):1479-86.

20. Cao Z, Wu X, Yen L, Sweeney C, Carraway KL. 3rd. Neuregulin-induced ErbB3 downregulation is mediated by a protein stability cascade involving the E3 ubiquitin ligase Nrdp1. Mol Cell Biol. 2007;27(6):2180-8.

21. Huang Z, Choi BK, Mujoo K, Fan X, Fa M, Mukherjee S, et al. The E3 ubiquitin ligase NEDD4 negatively regulates HER3/ErbB3 level and signaling. Oncogene. 2015;34(9):1105-15.

22. Mujoo K, Choi BK, Huang Z, Zhang N, An Z. Regulation of ERBB3/HER3 signaling in cancer. Oncotarget. 2014;5(21):10222-36.

23. Chen L, Siddiqui S, Bose S, Mooso B, Asuncion A, Bedolla RG, et al. Nrdp1-mediated regulation of ErbB3 expression by the androgen receptor in androgen-dependent but not castrate-resistant prostate cancer cells. Cancer Res. 2010;70(14):5994-6003.

24. Carraway KL 3. E3 ubiquitin ligases in ErbB receptor quantity control. Semin Cell Dev Biol. 2010;21(9):936-43. rd. .

25. Poller DN, Spendlove I, Baker C, Church R, Ellis IO, Plowman GD, et al. Production and characterization of a polyclonal antibody to the c-erbB-3 protein: examination of c-erbB-3 protein expression in adenocarcinomas. J Pathol. 1992;168(3):275-80.

26. Muller-Tidow C, Diederichs S, Bulk E, Pohle T, Steffen B, Schwable J, et al. Identification of metastasis-associated receptor tyrosine kinases in non-small cell lung cancer. Cancer Res. 2005;65(5):1778-82.

27. Yi ES, Harclerode D, Gondo M, Stephenson M, Brown RW, Younes M, et al. High c-erbB-3 protein expression is associated with shorter survival in advanced non-small cell lung carcinomas. Mod Pathol. 1997;10(2):142-8.

28. Yonesaka K, Hirotani K, Kawakami H, Takeda M, Kaneda H, Sakai K, et al. Anti-HER3 monoclonal antibody patritumab sensitizes refractory non-small cell lung cancer to the epidermal growth factor receptor inhibitor erlotinib. Oncogene. 2016;35(7):878-86.

29. Huang S, Li C, Armstrong EA, Peet CR, Saker J, Amler LC, et al. Dual targeting of EGFR and HER3 with MEHD7945A overcomes acquired resistance to EGFR inhibitors and radiation. Cancer Res. 2013;73(2):824-33.

30. Sun C, Hobor S, Bertotti A, Zecchin D, Huang S, Galimi F, et al. Intrinsic resistance to MEK inhibition in KRAS mutant lung and colon cancer through transcriptional induction of ERBB3. Cell Rep. 2014;7(1):86-93.

31. Noto A, De Vitis C, Roscilli G, Fattore L, Malpicci D, Marra E, et al. Combination therapy with antiErbB3 monoclonal antibodies and EGFR TKIs potently inhibits non-small cell lung cancer. Oncotarget. 2013;4(8):1253-65. 
32. Sequist LV, Waltman BA, Dias-Santagata D, Digumarthy S, Turke AB, Fidias P, et al. Genotypic and histological evolution of lung cancers acquiring resistance to EGFR inhibitors. Sci Transl Med. 2011;3(75):75ra26.

33. Engelman JA, Zejnullahu K, Mitsudomi T, Song Y, Hyland C, Park JO, et al. MET amplification leads to gefitinib resistance in lung cancer by activating ERBB3 signaling. Science. 2007;316(5827):1039-43.

34. Jaiswal BS, Kljavin NM, Stawiski EW, Chan E, Parikh C, Durinck S, et al. Oncogenic ERBB3 mutations in human cancers. Cancer Cell. 2013;23(5):603-17.

35. Kiavue N, Cabel L, Melaabi S, Bataillon G, Callens C, Lerebours F, et al. ERBB3 mutations in cancer: biological aspects, prevalence and therapeutics. Oncogene. 2020;39(3):487-502.

36. Littlefield P, Liu L, Mysore V, Shan Y, Shaw DE, Jura N. Structural analysis of the EGFR/HER3 heterodimer reveals the molecular basis for activating HER3 mutations. Sci Signal. 2014;7(354):ra114.

37. Wolff AC, Hammond MEH, Allison KH, Harvey BE, Mangu PB, Bartlett JMS, et al. Human Epidermal Growth Factor Receptor 2 Testing in Breast Cancer: American Society of Clinical Oncology/College of American Pathologists Clinical Practice Guideline Focused Update. J Clin Oncol. 2018;36(20):210522.

38. cBioPortal. cBioPortal for Cancer Genomics, 2019 [Available from: https://www.cbioportal.org.

39. Dry JR, Pavey S, Pratilas CA, Harbron C, Runswick S, Hodgson D, et al. Transcriptional pathway signatures predict MEK addiction and response to selumetinib (AZD6244). Cancer Res. 2010;70(6):2264-73.

40. Koutsopoulos AV, Mavroudis D, Dambaki KI, Souglakos J, Tzortzaki EG, Drositis J, et al.

Simultaneous expression of c-erbB-1, c-erbB-2, c-erbB-3 and c-erbB-4 receptors in non-small-cell lung carcinomas: correlation with clinical outcome. Lung Cancer. 2007;57(2):193-200.

41. CsToth I, Anthoine G, Berghmans T, Mascaux C, Paesmans M, Sculier JP, et al. C-erbB-3 expression in non-small cell lung cancer (NSCLC) patients treated by Erlotinib. Anticancer Res. 2011;31(1):281-5.

42. Lai WW, Chen FF, Wu MH, Chow NH, Su WC, Ma MC, et al. Immunohistochemical analysis of epidermal growth factor receptor family members in stage I non-small cell lung cancer. Ann Thorac Surg. 2001;72(6):1868-76.

43. Fontanini G, De Laurentiis M, Vignati S, Chine S, Lucchi M, Silvestri V, et al. Evaluation of epidermal growth factor-related growth factors and receptors and of neoangiogenesis in completely resected stage I-IIIA non-small-cell lung cancer: amphiregulin and microvessel count are independent prognostic indicators of survival. Clin Cancer Res. 1998;4(1):241-9.

44. Kumagai T, Tomita Y, Nakatsuka SI, Kimura M, Kunimasa K, Inoue T, et al. HER3 expression is enhanced during progression of lung adenocarcinoma without EGFR mutation from stage 0 to IA1. Thorac Cancer. 2018;9(4):466-71.

45. Cancer Genome Atlas Research N. Comprehensive molecular profiling of lung adenocarcinoma. Nature. 2014;511(7511):543-50. 
46. Cappuzzo F, Toschi L, Domenichini I, Bartolini S, Ceresoli GL, Rossi E, et al. HER3 genomic gain and sensitivity to gefitinib in advanced non-small-cell lung cancer patients. $\mathrm{Br} \mathrm{J}$ Cancer. 2005;93(12):1334-40.

47. Rouquette I, Lauwers-Cances V, Allera C, Brouchet L, Milia J, Nicaise $Y$, et al. Characteristics of lung cancer in women: importance of hormonal and growth factors. Lung Cancer. 2012;76(3):280-5.

48. Pellegrini C, Falleni M, Marchetti A, Cassani B, Miozzo M, Buttitta F, et al. HER-2/Neu alterations in non-small cell lung cancer: a comprehensive evaluation by real time reverse transcription-PCR, fluorescence in situ hybridization, and immunohistochemistry. Clin Cancer Res. 2003;9(10 Pt 1):3645-52.

49. Langer CJ, Stephenson P, Thor A, Vangel M, Johnson DH, Eastern Cooperative Oncology Group S. Trastuzumab in the treatment of advanced non-small-cell lung cancer: is there a role? Focus on Eastern Cooperative Oncology Group study 2598. J Clin Oncol. 2004;22(7):1180-7.

50. Sigismund S, Avanzato D, Lanzetti L. Emerging functions of the EGFR in cancer. Mol Oncol. 2018;12(1):3-20.

\section{Figures}


A
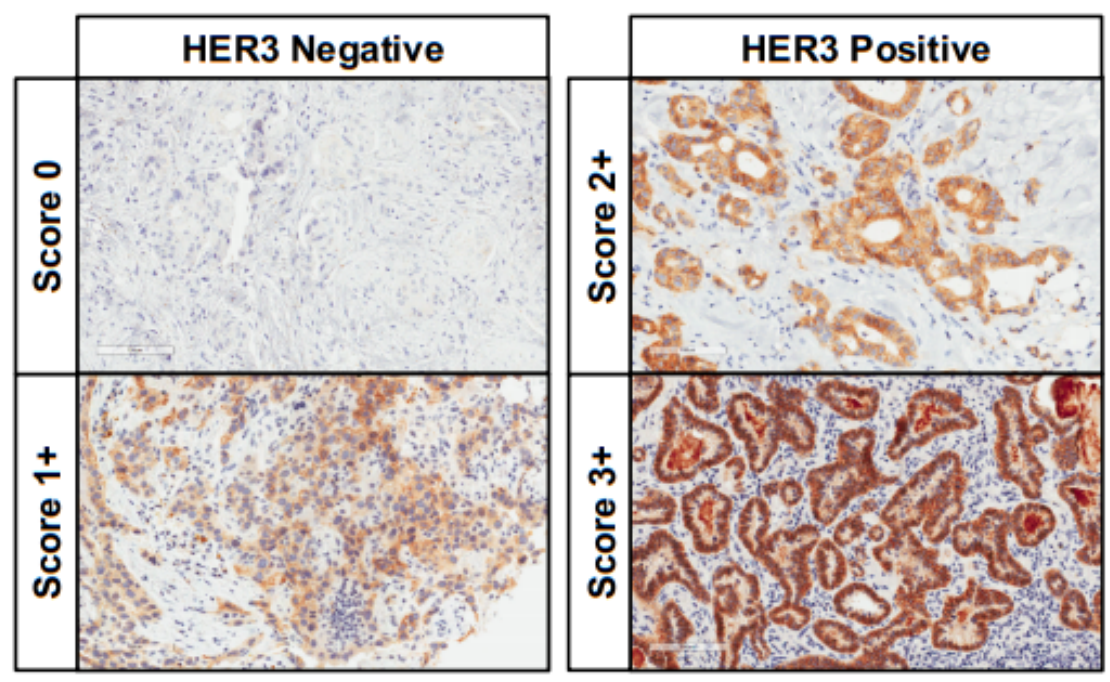

B

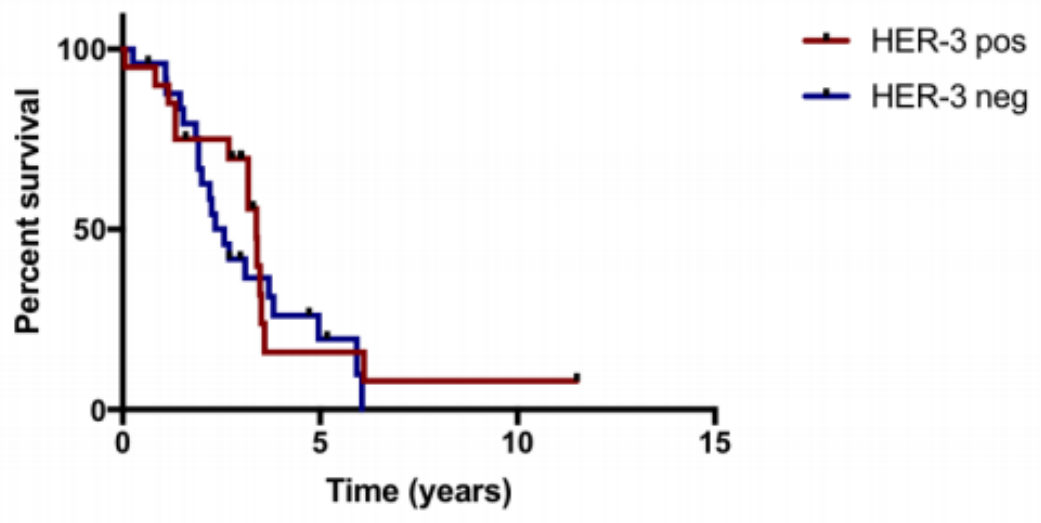

C

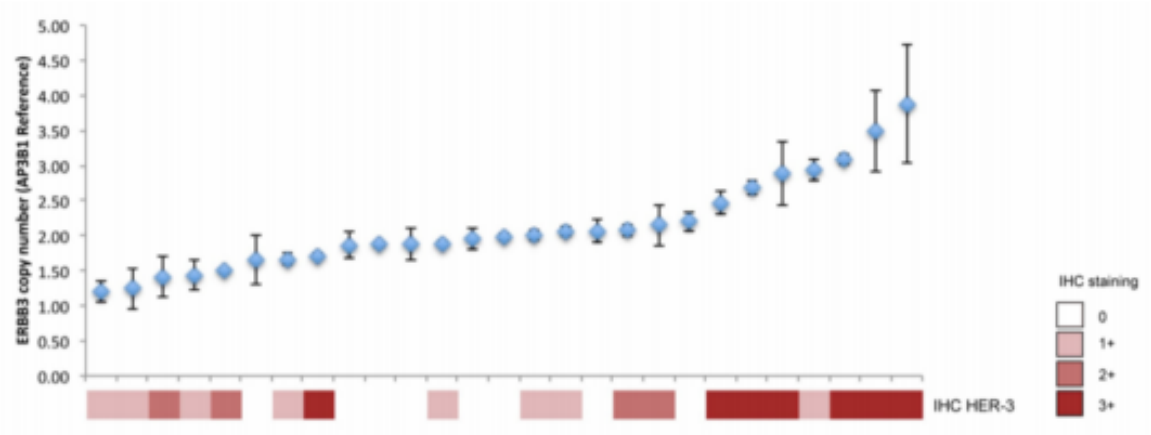

\section{Figure 1}

Adeno-NSCLC patient samples. Panel of HER-3 negative cases (0-1+) and HER-3 positive cases (2-3+) (A), Kaplan-Meir curve of HER-3 positive versus HER-3 negative cases (B), ERBB3 gene copy number and HER3 IHC (C) AP3B1; Adaptor Related Protein Complex 3 Subunit Beta 1, ERBB3: Erb-B2 Receptor Tyrosine Kinase 3, HER-3: receptor tyrosine-protein kinase erbB-3, IHC: immunohistochemistry, NSCLC: non-small cell lung cancer 


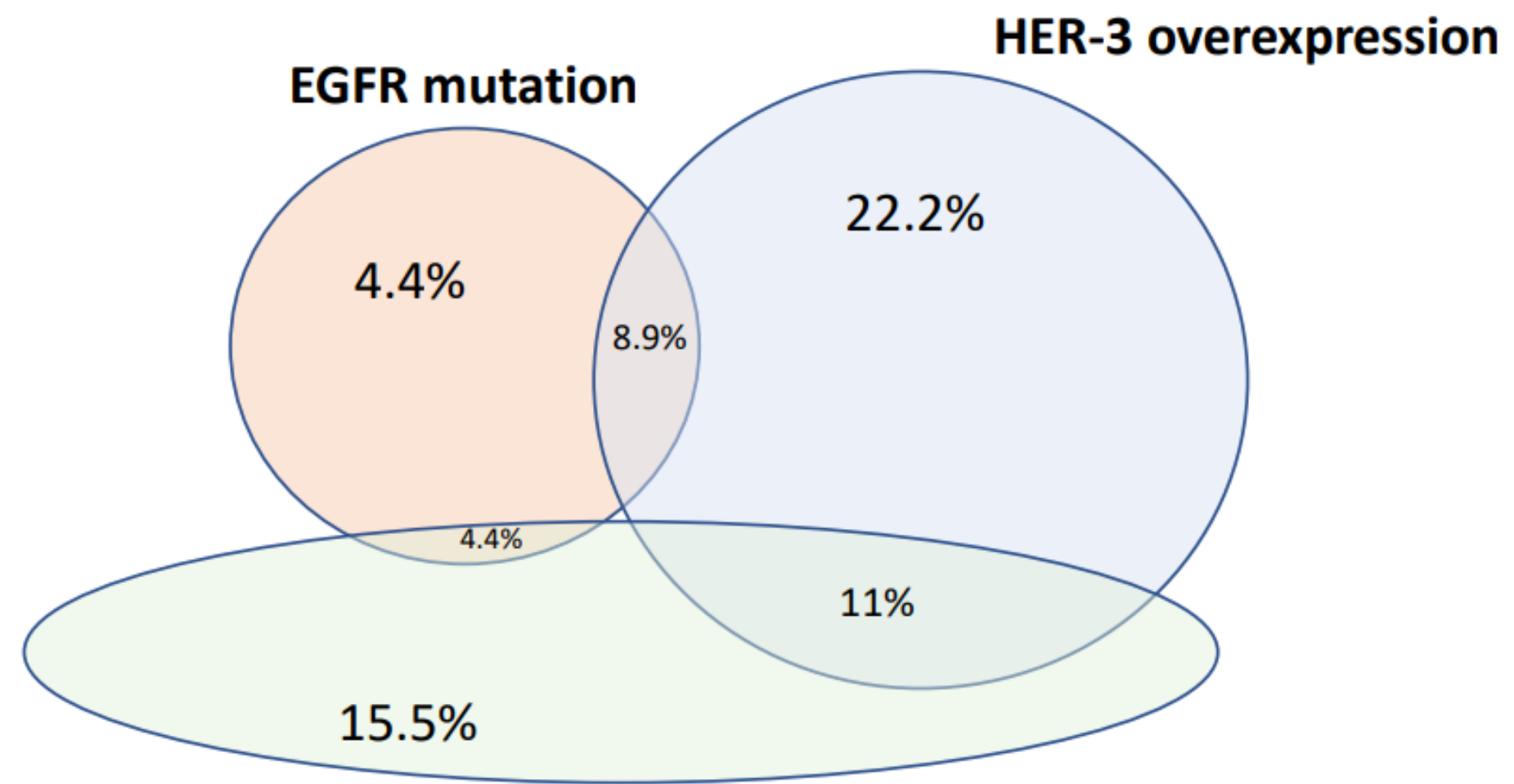

HER-2 overexpression

Figure 2

Frequency of aberrations in NSCLC as percentage of total population EGFR: epidermal growth factor receptor, ERBB3: Erb-B2 Receptor Tyrosine Kinase 3, HER-3: receptor tyrosine-protein kinase erbB-3; NSCLC: non-small cell lung cancer 
A

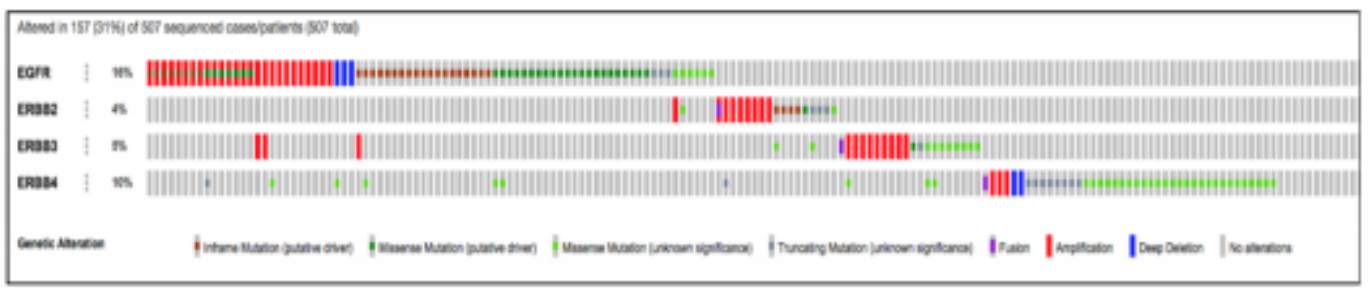

B

\section{C}
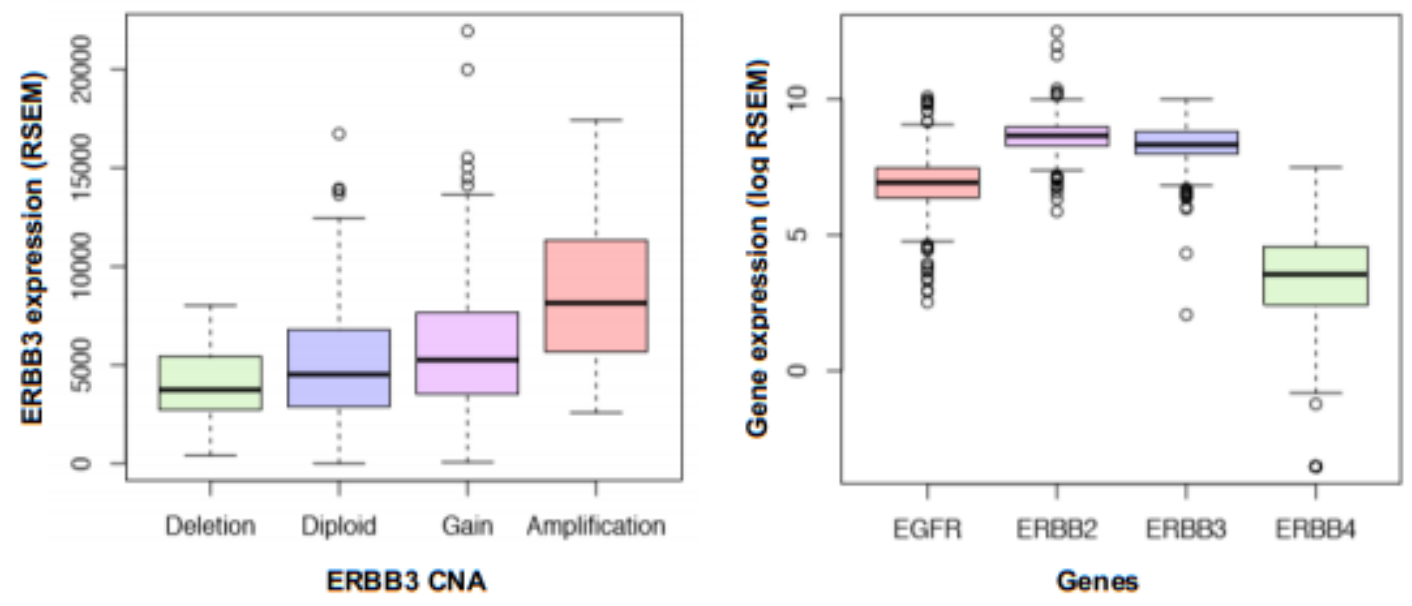

D

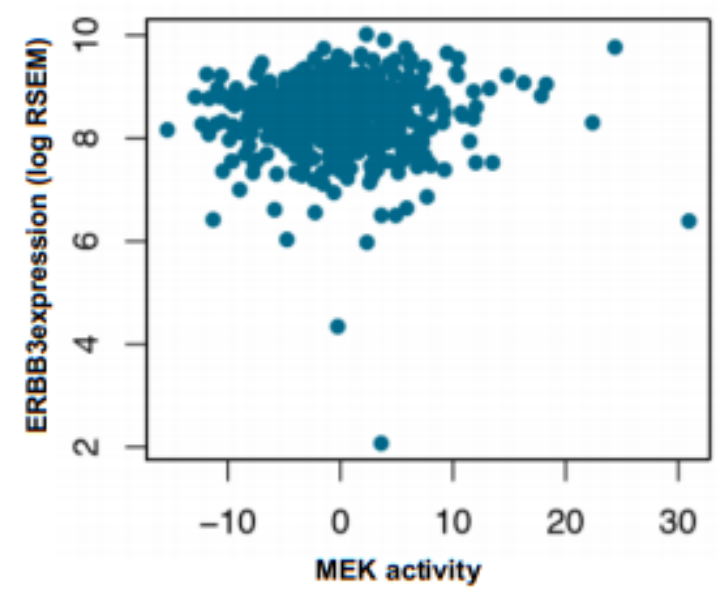

Figure 3

Adeno-NSCLC TCGA. Frequency of HER family genetic alterations (A); ERBB3 RNA expression with ERBB3 CNA (B), Gene expression of EGFR, ERBB2, ERBB3, and ERBB4 (C); Correlation of ERBB3 expression level with MEK activity (D). CNA: copy number aberration, EGFR: epidermal growth factor receptor, ERBB3: ErbB2 Receptor Tyrosine Kinase 3, HER-3: receptor tyrosine-protein kinase erbB-3; NSCLC: non-small cell lung cancer, TCGA: The Cancer Genome Atlas 


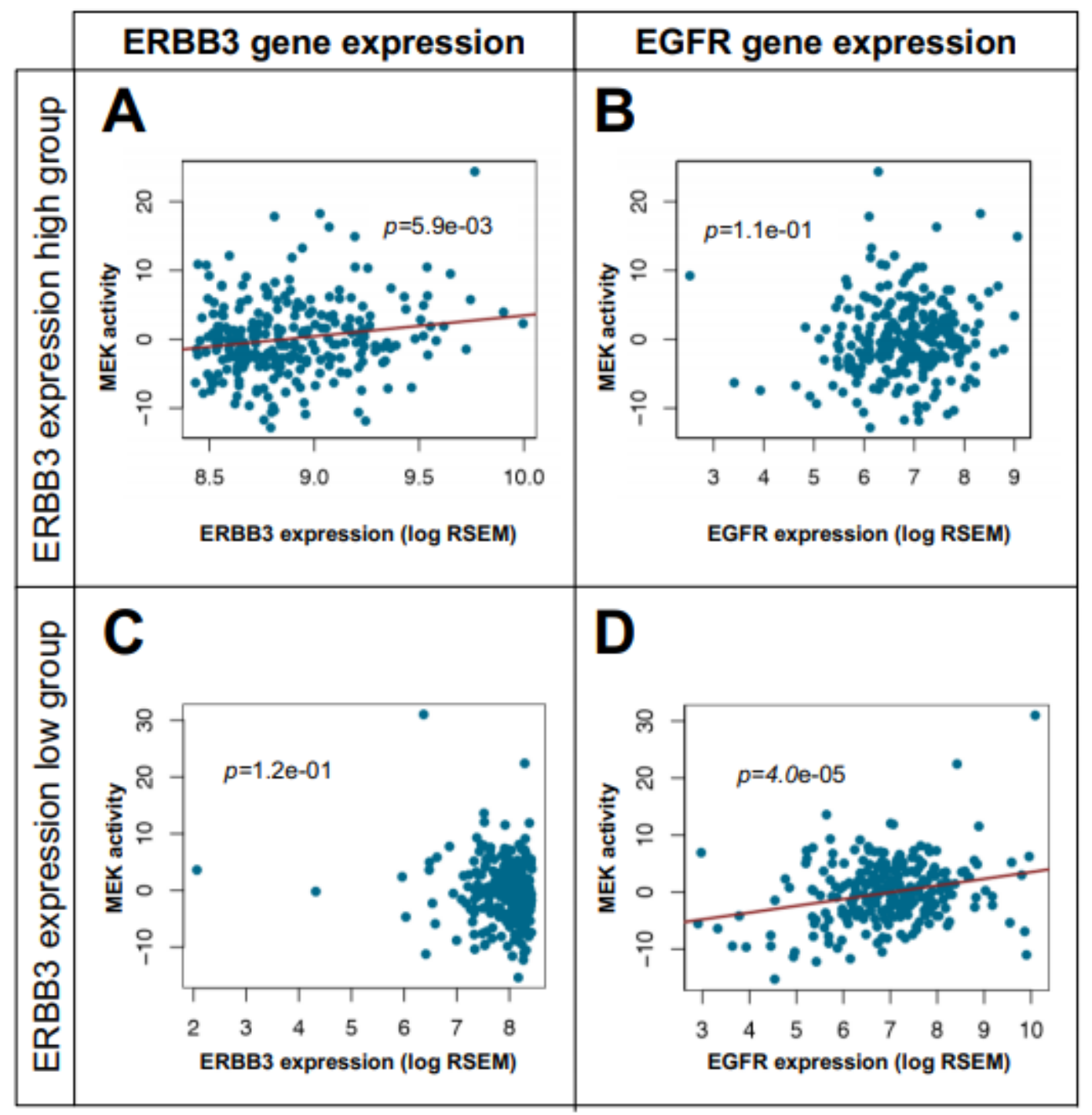

Figure 4

Correlation of gene expression with MEK activity in adeno-NSCLC TCGA. Correlation of ERBB3 expression level with MEK activity in ERBB3 expression high cancers (A), Correlation of ERBB3 expression level with MEK activity in ERBB3 expression low cancers (C). Correlation of EGFR expression with MEK activity in ERBB3 expression high cancers (B), correlation of EGFR expression with MEK activity in ERBB3 expression low cancers (D) HER-3: receptor tyrosine-protein kinase erbB-3, MEK: Mitogen-activated protein kinase kinase, NSCLC: non-small cell lung cancer, RSEM: RNA-Seq by Expectation Maximization

\section{Supplementary Files}

This is a list of supplementary files associated with this preprint. Click to download.

- HER3.SupplementaryMethodsMolCan.docx

- figS1.png 